Asian Journal of Engineering and Applied Technology

ISSN 2249-068X Vol.8 No.2, 2019, pp. 63-70

(C) The Research Publication, www.trp.org.in

\title{
Boundary Extraction and Vessel Width Calculation in Retinal Fundus Images
}

\author{
R. Manjunatha ${ }^{1^{*}}$ and H. S. Sheshadri ${ }^{2}$ \\ ${ }^{1}$ Assistant Professor, ${ }^{2}$ Professor, \\ ${ }^{1 \& 2}$ Department of Electronics and Communication Engineering, P.E.S. College of Engineering, Mandya, Karnataka, India \\ *Corresponding Author \\ E-Mail: rmanjunatha@gmail.com
}

\begin{abstract}
A retinal vessel width measurement algorithm is presented towards ROP (Retinopathy of Prematurity) plus diagnostic automation. The algorithm involving geometrical feature extraction with the image processing is used to compute the effective width of the major vessels in a retinal image. Width measurement is shown to be a statistical parameter estimation related to the statistics of the retinal information. The algorithm is applied to the generic data bases available and the results are found to be satisfactory with ophthalmologist opinion. The effectiveness of the algorithm depends on the fundus image capturing settings.

Keywords: OD, OPBA, RI, ROP, Tortuosity, Vascular Network
\end{abstract}

\section{INTRODUCTION}

ROP plus in infants is becoming a major threat, early diagnosis and treatment will help the infants to regain the normal vision. Diagnosis being a critical factor in deciding the impact and severity level, ophthalmologist needs a trustable diagnostic method for this. The ROP plusis identified by the tortuosity and dilation of the retinal vessels, its diagnostic requires careful study of vascular structure in a retinal image (RI). To make it effective and reliable Image processing based methods and algorithms have been developed.

Xiayu Xu et al., [2] presented a method to characterize vessel width based on arteries and veins classification, the accuracy of the method depends on the classifier, Faraz Oloumi et al.,[4] presented Gabour filter based MTA tracking and width measurement, where in the width is measured as two times the boundary distance, the accuracy depends on distance mapping. Gaussian approximation and morphological filtering based technique for segmenting vascular skeleton and characterizing vessels was presented by Heneghan et al., [7].

In spite of several methods available, still there is a depth to be covered in improvising accuracy of prediction with respect to the ROP levels categorization. In this context, this paper presents a novel analytical geometry based method with a statistical approach for the effective dilation measurement and analysis, being a decisive factor in ROP.The method is simulated and tested with the current standard data bases like Fire, Drive and Local data base.The results are consistent with respect to the traditional ophthalmologist verification, and further the method has been tested with the confusion matrix approach.

The contents of the paper are organized as: section II highlights the algorithm with a brief emphasis on image enhancement and preprocessing. This is followed by the discussion of vascular network, vessel extraction and major vessel identification process in section III. Section IV opens with the dilation measurement and allied issues while ends with the qualitative discussion of the statistical solution. Finally section $\mathrm{V}$ presents the results and analysis of the method, while leaving with the effectiveness and challenges in conclusion section VI.

\section{RETINAL IMAGE PRE-PROCESSING}

The RI contains anatomical and physical information about the retina in terms of geometrical objectives like optic disc, arteries and veins presented in 2D X 3 matrix. Out of this the dilation analysis requires only arteries and veins information. The task of analysis can be made simple by preprocessing the RI image for the vascular structure, independent of optic disc and color intensity information. Fig.1 shows the sequence of processes followed in preprocessing.

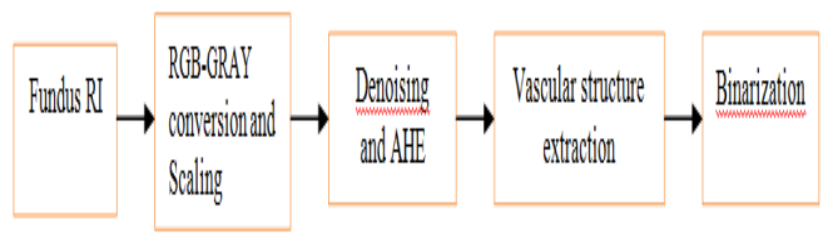

Fig. 1 Chain of pre-processing procedures adopted to prepare the RI images

Pre-processing starts with the conversion of colour fundus image into gray scale image, by translating the 2D X 3 channel RGB matrix, Figure 2a into 2D X 1 channel gray image, Fig.2b. In succession the gray image is normalized, denoised and subjected to adoptive histogram equalization (AHE) was applied to eliminate the noise and intensity variation effects. This was followed with binarization, Fig.2c for vascular structure extraction to get the vessel (Arteries and veins) network independent of optic disk $(\mathrm{OD})$, for the vessel dilation analysis. Overall pre- 
processing can be viewed as mathematical transformation of the image data from RGB domain to the binary domain as outlined in equation 1 .
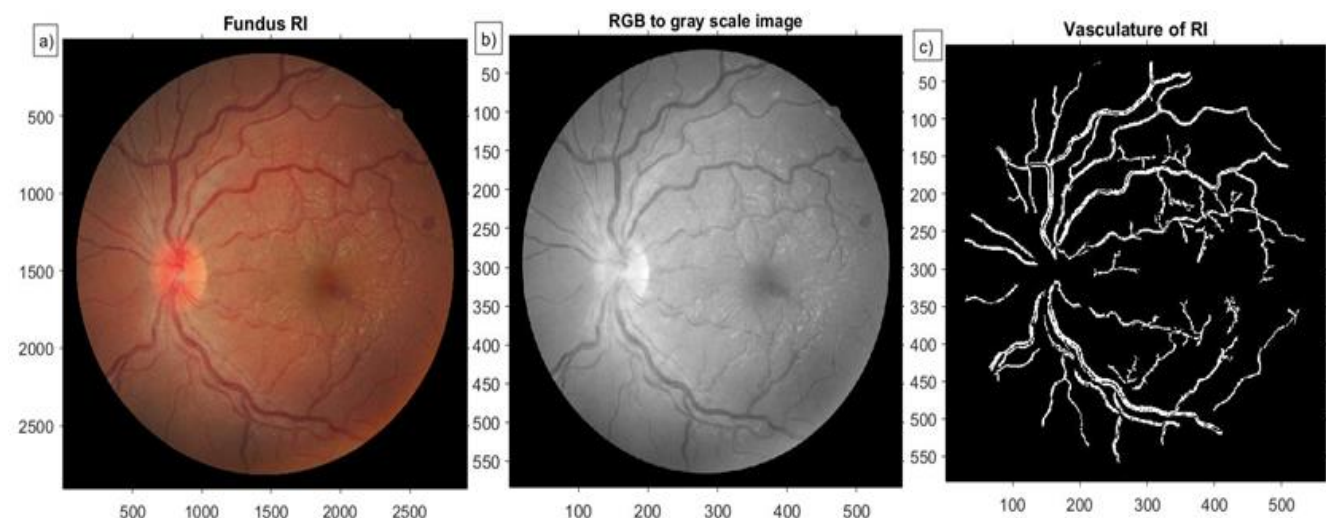

Fig. 2 a) Fundus retinal image b) Normalized grey scale image c) Vascular structure

First transformation converts a 2D X 3 Channel matrix into 2D X 1 channel matrix. Each pixel in 2D X 3 Channel matrix is defined with three components $R, G$ and $B$ after transformation each pixel is replaced with 8 bit gray scale value resulting in $2 \mathrm{D} \times 1$ matrix. Gray scale image is operated by switching function SW that switches the pixel value between 0 and 1 by comparing the pixel gray level with the threshold, which is set dynamically resulting in binarized image BI. Further, the switching also eliminates the OD information from the gray scale image, the binarized image $\mathrm{BI}$ can be interpreted as a matrix $V_{N}(x, y)$, (equation-1).

$$
\begin{aligned}
& G I=A H E\{R G B \rightarrow \operatorname{Gray}(R I)\} \\
& B I=S W\{G I\}, V_{N}(x, y)=B I
\end{aligned}
$$

Where RI - Retinal Image, BI- Binary image, SWswitching function

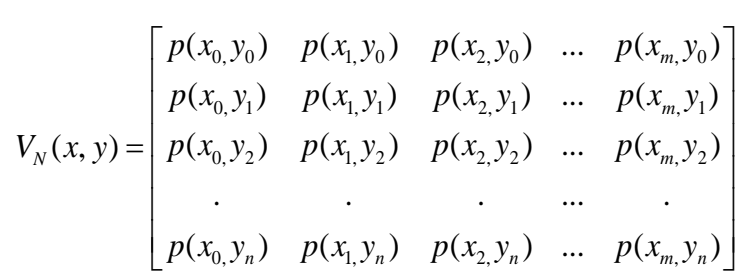

\section{VESSEL EXTRACTION AND MAJOR VESSEL IDENTIFICATION}

The preprocessed binarized image containing vascular network information, $V_{N}(x, y)$ matrix with the binarized pixel values is ready for vessel extraction. Analysis of the vascular network $V_{N}(x, y)$ shows that the vessels are connected pixel lines or regions with pixel value of 1 and having well defined Cartesian co-ordinate2-D positional information.

The background pixels that are not part of any vessels are set to zero value with image contrast enhancement and binarization as discussed in section II.
To extract the individual vessels, $\mathbf{V}$ (equation-2) from the network, $V_{N}(x, y)$ multi stage connectivity and regional property identification methods are employed repeatedly followed by correlation analysis and radon transform [10],the cuts and sprouts are treated as part of vessels.

$$
\mathbf{V}=\left\{V_{1}, V_{2}, V_{3} \ldots V_{\mathrm{n}}\right\}
$$

The dilation symptoms are most prevalently associated with the longer vessels due to the physiological process, chances of dilation being diagnosed with short length vessels are very meager [5]. This fact makes the selective vessel processing a natural choice.

The branching points in vascular network are treated as part of the vessel strings, vessels thus identified are subject to the pixel wise length analysis where in a longest vessel is selected and $75 \%$ of its value is taken as threshold for selecting the measurable vessels.

The vessel, $V_{i}$ with length (in terms of number of pixels) greater than the set threshold are classified as major vessels, $\mathbf{V}_{\mathbf{M}}$ (equation-3) and considered for further processing. Fig.3a shows the details of vessel structure, $\mathrm{MjV}$ - major vessels, Mn V-minor vessels and BP-branching point.

Fig.3b shows a major vessel extracted corresponding to vessel network of Fig.3a.

$$
\begin{aligned}
& \mathbf{V}_{\mathbf{M}}=\left\{V_{M 1}, V_{M 2}, V_{M 3} \ldots V_{M n}\right\} \\
& V_{M i} \in \mathbf{V}_{\mathbf{M}} \in \mathbf{V}_{\mathbf{N}}(x, y)
\end{aligned}
$$

The extracted vessels in $\mathbf{V}$ are independent geometrical sub sets, equation 4 with variant multi pixel width having well defined boundaries and spread at random angles with respect to the reference axis.

$$
V_{i}=\left\{p\left(x_{p}, y_{q}\right), p\left(x_{p}, y_{r}\right), p\left(x_{s}, y_{q}\right), \ldots p\left(x_{t}, y_{w}\right)\right\}
$$



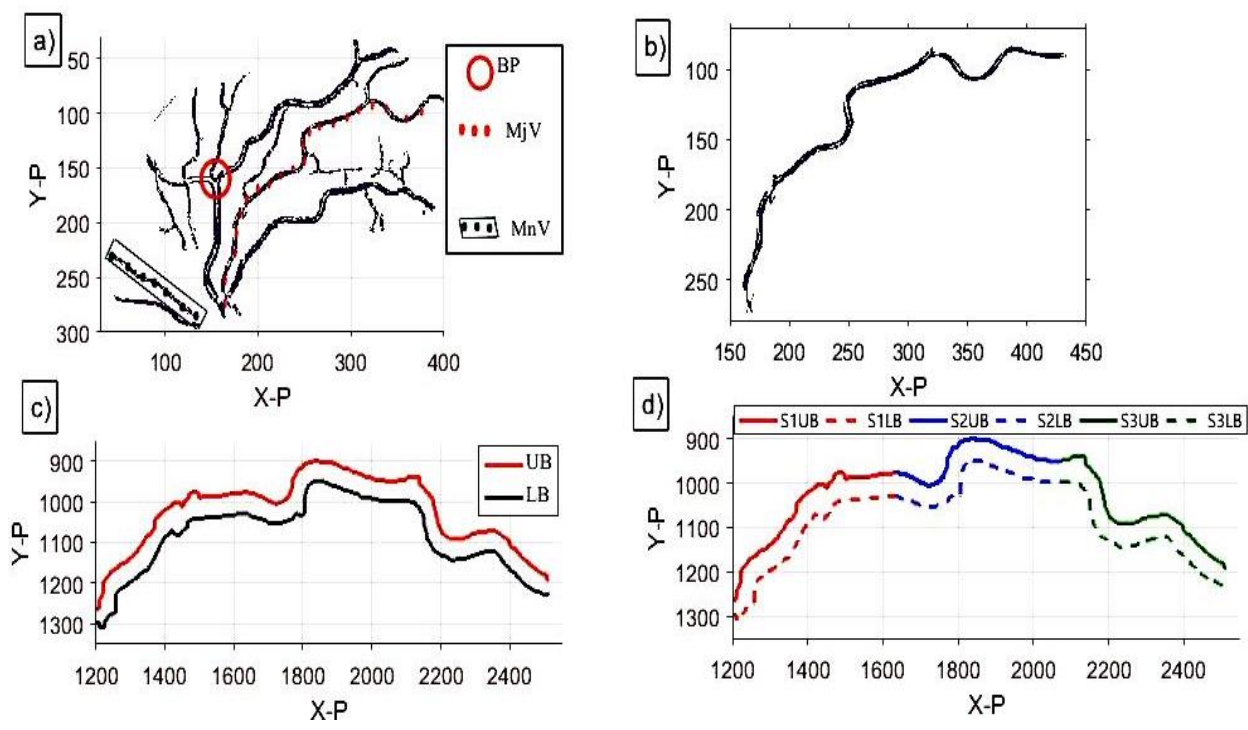

Fig. 3 (a) Part of a BI segment of Fig.2c (b) A major vessel extracted from BI of Fig.2c

(c) Upper and Lower boundary extracted for Fig.3b with $\theta$ rotation (d) Sample segmentation for Fig.3c

\section{BOUNDARY EXTRACTION AND SEGMENTATION}

Exact quantification of dilation in RI is a complex and nontrivial task, as RI consists of number of vessels (Arteries and viens) each having different length with continuous variation of width and direction [8]. Over and above this, the vascular network has number of branching points with Arteries and veins, hence making the measurement quite challenging.

Number of methods have been presented and reported for the retinal vessel dilation measurement. Zhou, Mark et al., [8] reported Gaussian fitting model based vessel tracking algorithm, where in segmentation technique was used to compute the width, center line and direction parameters of the vessel segment. Hunter et al., [6] computed the vessel width using 2-D Gaussian fitting of the vessel intensity and assuming center line of the vessel. The sigma (standard deviation) parameter of the differential Gaussian model was taken as vessel width. Heneghan et al., [7] reported the vessel width measurement by applying morphological and linear filtering to the fundus images and extracting binarized vascular structure. They computed vessel width by extending rotating reference line at every pixel of the segment, with every reference line rotation minimum distance was taken to be the width at the reference site pixel; a similar method was presented by G. Stabingis et al., [1].

The accuracy of these methods depends on Gaussian fitting and center point selection and they lack in addressing and accommodating the statistical and randomized distribution of geometrical features in vascular networks of RI. To fill in this gap by considering the statistical nature of the vascular pixel distribution information, sampled and effective averaging method is proposed here. a)

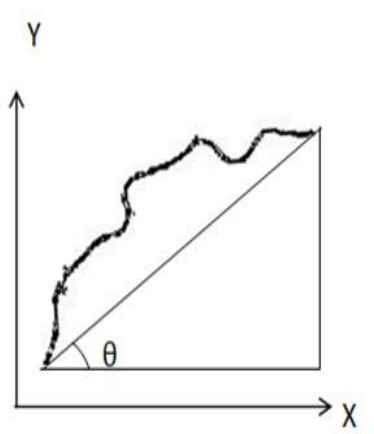

b)

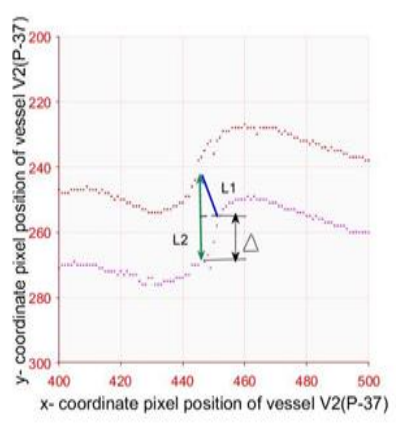

Fig. 4 a) Theta calculation plot for Fig.3b b) Error in the width measurement at a reference point $r$

In a vessel set at every $\mathrm{x}$ there are two extreme pixels $\mathrm{P}(\mathrm{x}$, $\left.\mathrm{y}_{\min }\right)$ and $\mathrm{P}\left(\mathrm{x}, \mathrm{y}_{\max }\right)$ along $\mathrm{y}$-axis that are part of upper and lower boundaries, defining the width of the vessel at that point, as shown in Fig.4(a). Thus by retaining maximum and minimum $\mathrm{Y}$-co-ordinate pixels at every $\mathrm{X}$-co-ordinate, all along the length of the vessel, boundaries UB (upper boundary) and LB (lower boundary) are extracted [11] i.e. outlined as algorithm in Fig. 5

For $m=1$ : length of vessel

theta $=\operatorname{COS}^{-1}(\mathrm{X} / \mathrm{hyp})$

$\operatorname{LB}\left(\mathrm{x}_{\mathrm{m}}, \mathrm{y}\right)=\min \left(\mathrm{V}_{\mathrm{M}}\left(\mathrm{x}_{\mathrm{m}}, \mathrm{y}\right)\right)$

$\mathrm{UB}\left(\mathrm{x}_{\mathrm{m}}, \mathrm{y}\right)=\max \left(\mathrm{V}_{\mathrm{M}}\left(\mathrm{x}_{\mathrm{m}}, \mathrm{y}\right)\right)$

end

The geometrical pattern of the vessel network is random with respect to the angular orientation of the vessels and this randomized angular orientation makes boundary extraction 
difficult and challenging. In order to overcome this, the vessels are angle compensated by rotation, angle of rotation $\boldsymbol{\theta}$ (equation-5) is computed as vessel curvature by considering either of both lateral boundaries.

$$
\boldsymbol{\theta}_{\mathbf{M}}=\left[\theta_{M 1}, \theta_{M 2}, \theta_{M 3} \ldots . \theta_{M n}\right]
$$

Where $\boldsymbol{\theta}_{\mathbf{M}}$ the angle of vessels and $\mathrm{n}$ is the vessel number The longitudinal distribution of the vessels makes dilation a statistical parameter, which needs to be computed as average of the set of widths computed all along the length of the vessel. To achieve this, each major vessel, $V_{M i}$ is divided into $\mathrm{N}$ number of segments, $V_{S i}$ equation 6. Fig. 3d shows the segment samples S1, S2 and S3 obtained for the vessel in Fig. 3b. The number of segments, $\mathrm{N}$ is set based on variance of the $\mathrm{K}$ boundary pixel w.r.t the mean of the $\mathrm{K}$ samples that includes the extreme left and right pixel $\mathrm{Y}$ coordinate along with (K-2) number of intermediate pixel $\mathrm{Y}$ coordinate, $\mathrm{K}$ is selected based on length of the vessel. Larger the variance more the number of segments. A discrete look up table is used to select the $\mathrm{N}$ based on variance value.

$$
V_{S i}=\left\{V_{S 1}, V_{S 2}, V_{S 3} \ldots . . V_{S N}\right\}
$$

Vessel width cannot be measured directly as the difference between the $\mathrm{Y}$ coordinate boundaries of pixels at reference point; this is valid only if the vessel boundaries are aligned parallelly, in all the other cases. This direct measurement will be erroneous with $\Delta$ error due to the curvy nature of the vessel boundaries as shown with line $L_{2}$ inFigure $4 b$ to overcome this width measurement must take slope of the vessel into account, this makes the task computationally complexto overcome this, here Oscillating Pendulum Based Algorithm(OPBA) is proposed.

\section{A. Oscillating Pendulum Based Algorithm for Width Measurement}

The problem with the width measurement as point to point difference between the boundaries is slope of the boundary strings, which leads to $\Delta$ error. The method of extending line and rotating vector are computationally demanding and suffers from growth and angle of rotation issues. The width problem can be solved by imaging a virtual problem suspended from the reference pixel that oscillates between the points LBP and RBP, while tracing different heights $h$ with discrete angle $\theta$ steps as per pixel distance as shown in Fig. 5a. With this the distance can be measured for every discrete movement of pendulum between boundaries and shortest distance can be recorded for the next measurement the pendulum point of suspension is shifted to next pixel. Rest of the details is discussed in the following paragraph.

A virtual oscillating pendulum based algorithm is introduced here for measuring the shortest Euclidian distance between the boundaries of the vessel at a sampled reference point in a segment. This algorithm is applied repetitively for every pixel in the boundary of the segment as shown in flowchart of Fig. 6, further the process is applied repeatedly for all the segments of a vessel. This is followed by the segment average vessel width and overall average of the vessel width computation, as discussed earlier (equations 8-10).

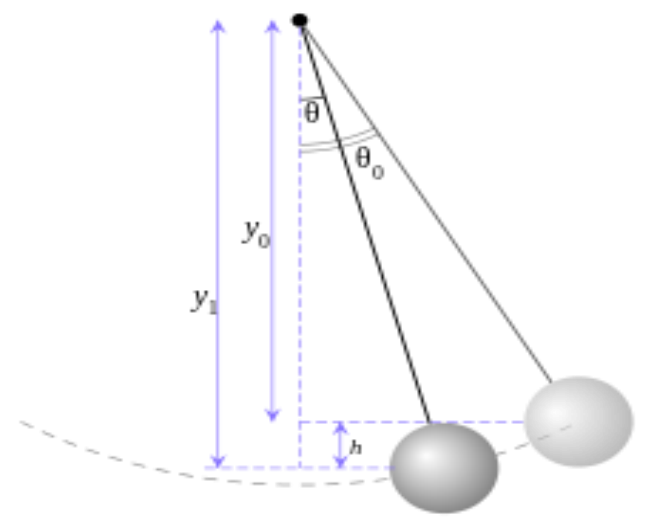

Fig. 5 a) OPB segment width computations

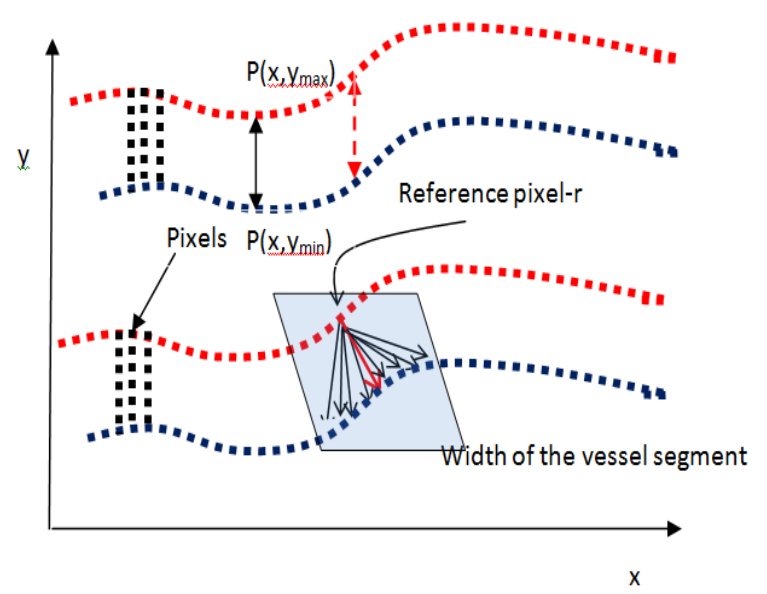

Fig. 5 b) Part of vessel segment in Fig. 3c

A reference pixel, $V_{\text {Siub }}\left(x_{r}, y_{r}\right)$ is selected from the upper string boundary of the vessel, $V_{\text {Siub }}$ in particular segment, a region of pixels, $R$ in the lower string boundary, $V_{\text {Silb }}$ centered at a pixel located exactly opposite the reference pixel is selected (equation 7). The region is considered with equal pixels on left and right side of the center pixel. Region with 9 pixels is found to be effective, smaller the region better the accuracy. Distance metric is computed between the reference pixel and all the pixels, $V_{\text {Silb }}\left(x_{j}, y_{j}\right)$ of the region (equation 8$)\left.W\right|_{(r, j)}$. The shortest among these is considering as the width at reference point is illustrating in Fig. 5b. The process is carried for every pixel in the segment and effectively over the length of the vessel.

Further to measure the width at any point selecting appropriate sample pixels in the boundary string is essential 
and it defines the accuracy of width measured in terms of number of pixels.

$$
\begin{aligned}
& V_{\text {Siub }}=P_{1}\left(x_{1}, y_{1 \text { max }}\right) P_{2}\left(x_{2}, y_{2 \max }\right) \ldots \ldots P_{n}\left(x_{n}, y_{\text {nmax }}\right) \\
& V_{\text {Silb }}=P_{1}\left(x_{1}, y_{1 \text { min }}\right) P_{2}\left(x_{2}, y_{2 \text { min }}\right) \ldots \ldots P_{n}\left(x_{n}, y_{n \text { min }}\right)
\end{aligned}
$$

As mentioned the width is Euclidian distance between the boundaries at reference point. The varying boundary curvature construction of vessels calls in for a shortest distance measurement for the width calculation.

$$
\left.W\right|_{(r, j)}=\min \left(\sqrt{V_{\text {Siub }}\left(x_{r}, y_{r}\right)^{2}-V_{\text {Silb }}\left(x_{j}, y_{j}\right)^{2}}\right), r-\frac{(R-1)}{2}<j<r+\frac{(R-1)}{2}
$$

r- Reference point w.r.t $\mathrm{X}$-axis and $\mathrm{R}$ is Number of pixels defining region.

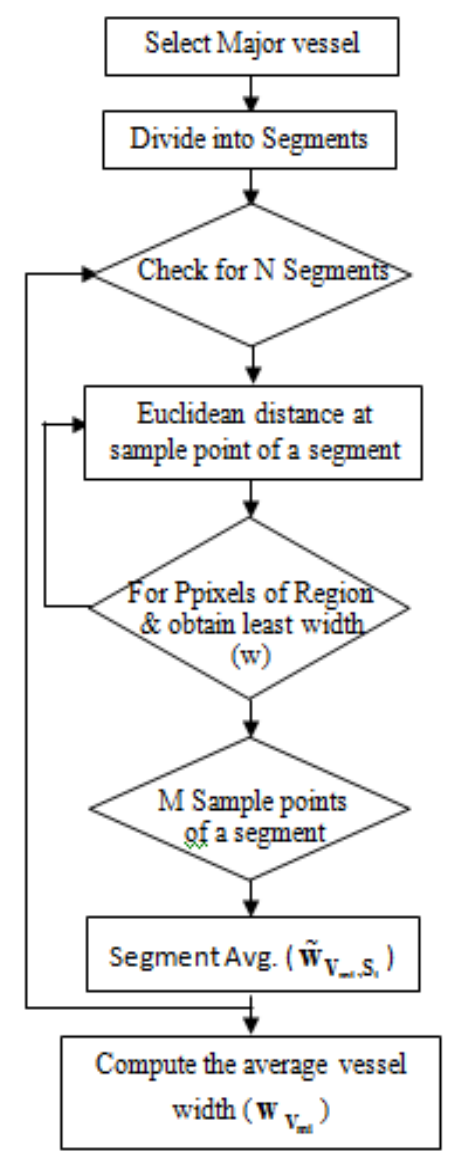

Fig. 6 Flow Chart of width computation

\section{B. Effective Width Computation}

This paper models the width measurement as Euclidian distance between two set of two valued functions that corresponds to the vessel boundaries. The vessel boundaries are extracted from the binarized retinal image by applying cross sectional max min technique of sorting out pixels in extracted vessel image. This boundary based method of vessel width computation combined with statistical computation approach reduces the computation complexities associated with the extending reference line methods of [2], [4], [9] and also overcomes the inaccuracies of vessel tracking algorithms [8].

Measuring the vessel width i.e. boundary to boundary distance at any sample point of a vessel image is a peculiar job due to the difference in curvature of the boundaries.

Keeping in view of the temporal distribution of the vessels width is estimated as average effective width of the vessel, $w_{V_{m i}}$ rather than a single point of measure.

Each of the vessels is divided into $\mathrm{N}$ number of segments of

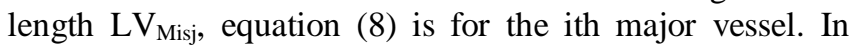
every segment width is measured at each pixel site of the vessel, as shown in Fig.7 with labels W1S1, W2S1 etc representing width measured at reference points 1 and 2 in segment S1.As pointed earlier length of segment is set selected based on the overall vessel length, so as to reduce the width error due statistical variations. For every $j^{\text {th }}$ segment one average width, $\tilde{w}_{V_{m i}, S_{j}}$ is computed, with $\mathrm{S}_{\mathrm{j}}$ number of segments resulting in $S_{j}$ number of average widths per vessel, refer equation (9).

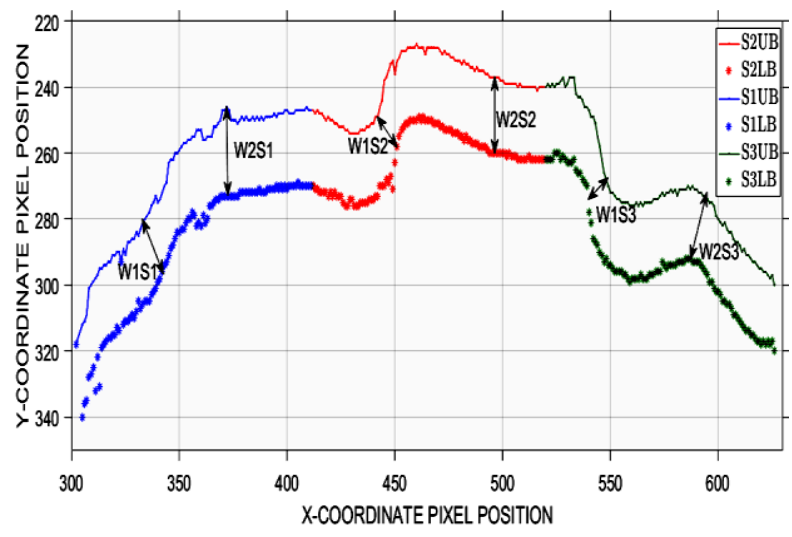

Fig. 7 Vessel widths at different segment pixel position

$$
\tilde{w}_{V_{m i}, S_{j}}=\frac{\sum_{n=1}^{S_{s, V_{m i}}} w_{n}}{S_{s, V_{m i}}}
$$

Where $S_{s}$ is segment size

$$
w_{V_{m i}}=\frac{\sum_{j=1}^{M_{V_{m i}}} \tilde{w}_{V_{m i}, S_{j}}}{N_{V_{m i}}}
$$

Consequently the segment width averages are normalized with respect to number of segments yielding effective width, equation (10). 


\section{RESULTS AND DISCUSSION}

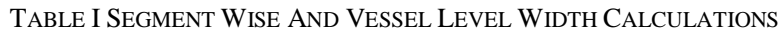

\begin{tabular}{|c|c|c|c|c|c|c|}
\hline & $\tilde{w}_{V_{1}, S_{j}}$ & $\tilde{w}_{V_{2}, S_{j}}$ & $\tilde{w}_{V_{3}, S_{j}}$ & $\tilde{w}_{V_{4}, S_{j}}$ & $\tilde{w}_{V_{5}, S_{j}}$ & $\tilde{w}_{V_{6}, S_{j}}$ \\
\hline Data-Set & V1 & $\mathbf{V 2}$ & V3 & V4 & V5 & V6 \\
\hline $\begin{array}{l}\text { P_37-FIRE } \\
\text { Avg. width at each segment level }\end{array}$ & $\begin{array}{l}19.7796 \\
15.9506 \\
11.7006 \\
10.2138 \\
14.6217 \\
13.7401 \\
10.4967 \\
10.3092\end{array}$ & $\begin{array}{l}12.7515 \\
11.5575 \\
08.0727 \\
11.2363 \\
07.9878 \\
11.4969 \\
08.5212 \\
09.1363\end{array}$ & $\begin{array}{l}13.1350 \\
10.5258 \\
11.8534 \\
10.8074 \\
10.6063 \\
15.1580 \\
09.7902 \\
09.4942\end{array}$ & $\begin{array}{c}9.5861 \\
8.7083 \\
9.2139 \\
10.2801 \\
9.7305 \\
10.2027 \\
7.9305 \\
7.3055\end{array}$ & $\begin{array}{l}26.1821 \\
21.2178 \\
13.2375 \\
17.8425 \\
10.1178 \\
10.1464 \\
10.2892 \\
09.5857\end{array}$ & --- \\
\hline Avg. vessel width $\left(w_{V_{m i}}\right)$ & $\mathrm{V} 1=13.35$ & $\mathrm{~V} 2=10.09$ & $\mathrm{~V} 3=11.42$ & $\mathrm{~V} 4=9.11$ & $\mathrm{~V} 5=11.83$ & \\
\hline $\begin{array}{l}\text { P29_1-FIRE } \\
\text { Avg. width at each segment level }\end{array}$ & $\begin{array}{c}14.1675 \\
9.7280 \\
7.8653 \\
7.5027 \\
9.4972 \\
8.0851 \\
15.4917 \\
18.8296 \\
\end{array}$ & $\begin{array}{c}7.6529 \\
8.3246 \\
10.4813 \\
12.0709 \\
7.9962 \\
15.1977 \\
15.4589 \\
15.2537 \\
\end{array}$ & $\begin{array}{c}7.4522 \\
8.7316 \\
10.8786 \\
9.4816 \\
10.2683 \\
9.0992 \\
15.1654 \\
73.5735 \\
\end{array}$ & $\begin{array}{c}7.1925 \\
9.9020 \\
6.8412 \\
11.5642 \\
9.9831 \\
8.9425 \\
8.5979 \\
14.2770 \\
\end{array}$ & $\begin{array}{l}10.8304 \\
14.0584 \\
11.9298 \\
13.7777 \\
11.3508 \\
16.6666 \\
10.3216 \\
17.1842 \\
\end{array}$ & $\begin{array}{c}7.4778 \\
8.7500 \\
11.2057 \\
7.7816 \\
10.1171 \\
9.3829 \\
8.8322 \\
9.2468 \\
\end{array}$ \\
\hline Avg. vessel width $\left(w_{V_{m i}}\right)$ & $\mathrm{V} 1=11.39$ & $\mathrm{~V} 2=11.55$ & $\mathrm{~V} 3=18.08$ & $\mathrm{~V} 4=9.66$ & $V 5=13.26$ & $\mathrm{~V} 6=9.09$ \\
\hline $\begin{array}{l}\text { 11_test -DRIVE } \\
\text { Avg. width at each segment level }\end{array}$ & $\begin{array}{c}10.3736 \\
7.7387 \\
9.0870 \\
7.4410 \\
7.0927 \\
7.7724 \\
8.5252 \\
9.1348 \\
\end{array}$ & $\begin{array}{l}7.2532 \\
6.9476 \\
9.0524 \\
9.1528 \\
9.8821 \\
8.3755 \\
7.2576 \\
7.1550 \\
\end{array}$ & $\begin{array}{l}18.6006 \\
11.0941 \\
07.6655 \\
12.0422 \\
15.3019 \\
07.4642 \\
10.2500 \\
05.5584 \\
\end{array}$ & $\begin{array}{l}78.3484 \\
13.3962 \\
11.6835 \\
10.1143 \\
10.1303 \\
08.0665 \\
13.9069 \\
07.9734 \\
\end{array}$ & ---- & ---- \\
\hline Avg. vessel width $\left(w_{V_{m i}}\right)$ & $\mathrm{V} 1=8.39$ & $\mathrm{~V} 2=8.13$ & $\mathrm{~V} 3=10.99$ & $\mathrm{~V} 4=10.72$ & & \\
\hline $\begin{array}{l}\text { Im0236-STARE- } \\
\text { Avg. width at each segment level }\end{array}$ & $\begin{array}{c}10.0397 \\
12.2251 \\
17.7351 \\
12.9536 \\
9.8940 \\
13.0132 \\
15.9139 \\
21.8443 \\
\end{array}$ & $\begin{array}{c}14.7653 \\
10.5251 \\
9.9609 \\
14.3575 \\
11.2067 \\
10.7988 \\
10.0726 \\
13.662 \\
\end{array}$ & $\begin{array}{c}19.5457 \\
13.7408 \\
13.3201 \\
12.1128 \\
15.4298 \\
14.125 \\
13.5640 \\
10.3902 \\
\end{array}$ & ---- & --- & ---- \\
\hline Avg. vessel width $\left(w_{V_{m i}}\right)$ & $\mathrm{V} 1=14.20$ & $\mathrm{~V} 2=11.91$ & $\mathrm{~V} 3=14.02$ & & & \\
\hline
\end{tabular}

As outlined in the abstract, aim of the work is to quantify the vessel width. To accomplish this, sample images of the Drive, Fire and local data set are processed through the preprocessing steps and the proposed algorithm. Fig.2 shows the results of preprocessing applied to the sample retinal image from the database. Some of the authors have preferred to mention the vessel dimensions in terms of micro meter [4], here for the sake of brevity and generalization vessel dimensions are treated in terms of pixel count.

Fig.8a shows the binarized vascular structure for the retinal image P_37-FIRE from the fire data base. The vascular structure was processed for the lengthwise largest vessel identification and vessel V4 was identified as the largest vessel with length of 353 pixels. Following this a threshold of $75 \%$ of this length, i.e 265 was applied to identify the major vessels, V1-V5 as shown with labels in Fig. 8b.The number $75 \%$ has been set based on the iterative analysis of the retinal images for the longer vessel identification, any number less than this will also be fine but will lead to greater computational effort with approximately the same result.

The higher threshold value leads to dubious situation with computational efficiency with lesser vessels, in case of images with blurring and alignment issues. In continuation of this, vessels are processed for boundary identification followed by segmentation where in the vessel's are divided into 8 segments as discussed in section 3. Fig. 9 b shows the segments of boundary extracted vessel V2 with segment labels S1-S8. 

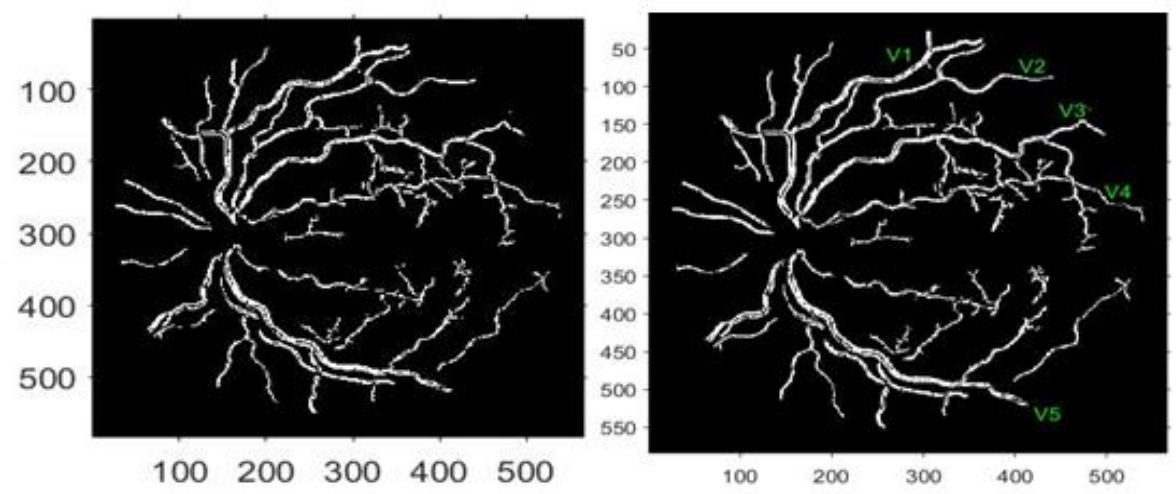

Fig. 8 a) Binarized image of Fire data set (P_37_1) b) Major vessels labeled for Fig.8a

Finally OPBA is applied over all the major vessels with identified segments, as highlighted in section 4. Table I shows the results of OPBA listed vessel wise with segment averages and vessel averages for the sample images of data bases as mentioned with labels. The results shows that the segment wise averages varies with $10-15 \%$ with respect to the vessel averages, this may be due to the digitization of the data and resolution of the camera. Further the widths computed here will have inaccuracy due to the camera orientation and capturing [3]. The fractional numbers are due to the averaging process adopted.

In table I the computations for the retinal image P_37-FIRE shows that the average vessel width measured and recorded to be varying from vessel to vessel for the same retinal image. This variation, as listed in table I is observed for all the cases. Case Im0236-STARE was found to be identified with only 3 major vessels; again this may be due to the threshold which was set to $75 \%$ of the longest vessel. Observing ate the results; the average vessel width in this case appears to be more consistent. The table I also shows, some abrupt segment averages as highlighted, this is due to the steep rise or fall of the vessel boundaries, Fig.9a shows the major vessel V4 for retinal image 11_test -DRIVE, first segment in the vessel has very steep fall. The steepness of the segments resulted in average segment width of 78.3484, which is large in comparison to other segments of the same vessel and segments of other vessels for the same image. This abrupt variation of the width can be eliminated by applying segment rotation.
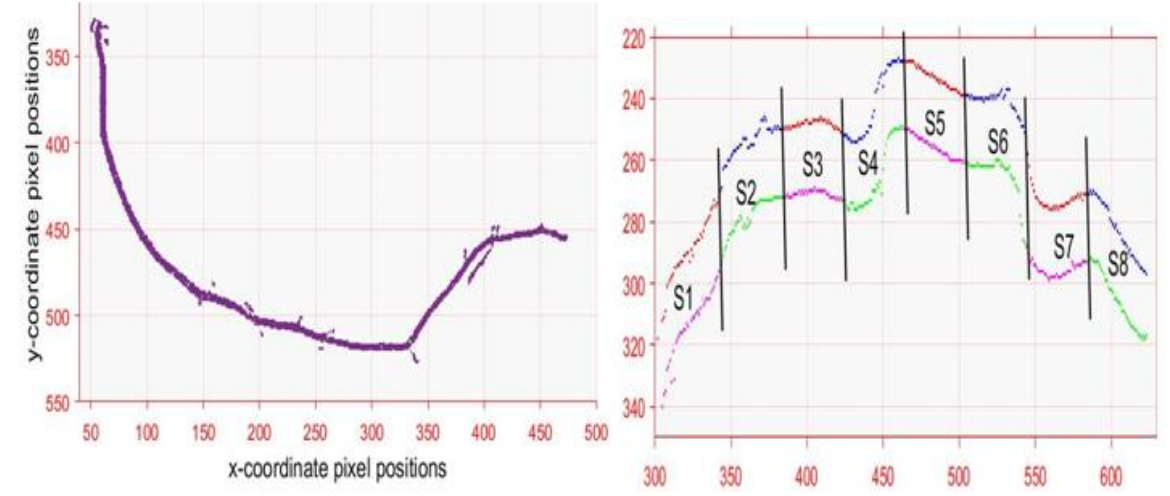

Fig. 9 a) Vessel V4 for retinal image 11_test -DRIVE b) Boundary extracted for V2 (P_37_1) vessel with segment labeled S1-S8

\section{CONCLUSION}

A novel approach for the retinal vessel width computation is proposed in the form of OPBA. Retinal vessel width measured with OPBA exhibits good accuracy in comparison to the earlier proposed algorithms as reported by Faraz Oloumi et al., [4] and Heneghan et al., [7]. Current OPBA algorithm suffers from slope error in some of the cases in vessels with abrupt slope variations. To overcome this, an extension to the current OPBA algorithm is planned for the future with segment rotation as feature.

\section{ACKNOWLEDGEMENT}

The authors are grateful to Dr. Satishkumar B V, Minto hospital, Bangalore for providing infant's images and also public data base Drive, Fire data set. Thanks to the VGST of Karnataka for having funded this project under medical image analysis laboratory at our institution.

\section{REFERENCES}

[1] Giedrius Stabingis, Jolita Bernataviciene, Gintautas Dzemyda, Alvydas Paunksnis, Povilas Treigys, Ramute Vaicaitiene, and Lijana 
Stabingien "Automatization of Eye Fundus Vessel Width Measurements", vipimage-2017, Springer International Publishing, published in 2018, DOI: 10.1007/978-3-319-68195-5_85.

[2] Meindert Niemeijer, Xiayu Xu, Alina V. Dumitrescu, Priya Gupta, Bram van Ginneken, James C. Folk, and Michael D. Abrámoff, "Automated Measurement of the Arteriolar-to- Venular Width Ratio in Digital Color Fundus Photographs", IEEE Transactions on Medical Imaging, Vol. 30, No. 11, Nov. 2011.

[3] G D. arway-Heath, A. Rudnicka, T. Lowe, P. Foster, F. Fitzke and R. Hitchings, "Measurement of optic disc size: equivalence of methods to correct for ocular magnification", Br. J. Ophthalmol, Vol. 82, 643649, 1998.

[4] Faraz Oloumi, Rangaraj M. Rangayyan, Anna L. Ells "Measurement of Vessel Width in Retinal Fundus Images of Preterm Infants with Plus Disease", IEEE International Symposium on Medical Measurements and Applications, MeMeA, 2014.

[5] Chisako Muramatsu, Yuji Hatanaka, Tatsuhiko Iwase, Takeshi Hara and Hiroshi Fujita "Automated detection and classification of major retinal vessels for determination of diameter ratio of arteries and veins", Medical Imaging 2010: Computer-Aided Diagnosis, edited by Nico Karssemeijer, Ronald M. Summers, Proc. of SPIE, Vol. 7624,76240J.

[6] J. Lowell, A. Hunter, D. Steel, A. Basu, R. Ryder and R. L. Kennedy, "Measurement of retinal vessel widths from fundus images based on
2-D modeling", IEEE Transactions on Medical Imaging, Vol. 23, No. 10, pp. 1196-1204, 2004.

[7] C. Heneghan, J. Flynn, M. O'Keefe and M. Cahill, "Characterization of changes in blood vessels width and tortuosity in retinopathy of prematurity using image analysis", Medical Image Analysis, Vol. 6, No. 1, pp. 407-429, 2002.

[8] Liang Zhou, Mark S. Rzeszotarski, Lawrence J. Singerman and Jeanne M. Chokreff "The Detection and Quantification of Retinopathy Using Digital Angiograms", IEEE Transactions on Medical Imaging, Vol. 13, No. 4, Dec. 1994.

[9] Ana Maria Mendonça and Aurélio Campilho, "Segmentation of Retinal Blood Vessels by Combining the Detection of Centerlines and Morphological Reconstruction", IEEE Transactions on Medical Imaging, Vol. 25, No. 9, Sept. 2006.

[10] Reza Pourreza, Touka Banaee et al., "A Radon Transform Based Approach for Extraction of Blood Vessels in Conjunctival Images", (C) Springer-Verlag Berlin Heidelberg, pp. 948 - 956, 2008.

[11] R Manjunatha, Mahesh Koti and Dr. H.S. Sheshadri "Boundary Extraction and Tortuosity Calculation in Retinal Fundus Images", Springer, pp. 1119-1130, PESCE, Mandya, published in April2019.Doi: https://doi.org/10.1007/978-981-13-5802-9_96.ICERECT2018 , 